positive and negative predictive values of International Ovarian Tumor Analysis (IOTA) - simple rules and risk of malignancy index (RMI) classification in differentiating benign and malignant adnexal masses.

Methods: This is a prospective comparative study performed in the Department of Obstetrics and Gynaecology at All India Institute of Medical Sciences, Jodhpur over 2 years from 2018 to 2020. Women with adnexal masses in the reproductive and postmenopausal age group, planned for surgery were included in the study. Those with known malignancies or already receiving treatment were excluded. A complete clinical assessment, cancer antigen 125 , and ultrasound scans were done along with other tumor markers and radiological investigations as per patient profile and consultant discretion. The findings were then classified as benign or malignant as per the IOTA simple rules. The RMI-2 score was also calculated for the same with a cut-off of 200 for benign masses. The results obtained were compared with the histopathology report obtained postoperatively.

Results: This study included 174 patients in total. IOTA—simple rules had an overall sensitivity of $96.67 \%$, specificity of $92.36 \%$, positive predictive value (PPV) of $72.5 \%$, negative predictive value (NPV) of $99.25 \%$. The scores were higher if indeterminate cases were excluded. RMI-2 had a sensitivity of $80 \%$, specificity of $82.64 \%$, PPV of $48.98 \%$ and $95.20 \%$ was NPV. Both of these scoring systems were then compared with each other and it concluded that IOTA simple rules have a higher sensitivity, specificity as compared to RMI. With an accuracy of $93.1 \%$. The cost-effectiveness of IOTA- simple rules makes it an efficient outpatient department (OPD) procedure of classifying adnexal masses.

Conclusion: We can conclude that IOTA-simple rules, can be used as an effective screening criterion for differentiating adnexal masses into benign and malignant on an OPD basis.

Poster (M10)

Miscellaneous

https://doi.org/10.3802/jgo.2021.32.S1.M10

\section{Perception of gynaecologic cancer patients undergoing surgical management at a tertiary care center during COVID-19 pandemic: a descriptive study}

\section{Sarita Kumari," Rudrika Chandra, Dipanwita Banerjee, Neerja Bhatla}

All India Institute of Medical Sciences, New Delhi, India (sarita2325@gmail.com)

Objective: Coronavirus disease 2019 (COVID-19) pandemic led to a major transformation of healthcare services across the globe regardless of economic status. Less is known about the perspective of oncology patients who underwent a major surgical procedure during this challenging period when protocols were being implemented.

Methods: We conducted a questionnaire-based survey on patients who underwent surgical procedure for a gynaecologic malignancy at All India Institute of Medical Sciences, New Delhi between March 2020-December 2020. The survey was carried out telephonically after verbal consent to assess their perspective regarding medical care during pandemic.

Results: Median age of 88 patients was 56 years (range, 18-74 years). Most common sites were ovary $(\mathrm{n}=62,70 \%)$ followed by endometrium ( $\mathrm{n}=21,24 \%)$. Only38 (43\%) participants were fully aware of COVID-19(cause/spread/symptoms/outcome) whereas $67(76 \%)$ participants felt scared of suffering from cancer if did not get timely surgical management. Out of all patients, 38 $(43 \%)$ felt comfortable in hospital visits, $36(41 \%)$ knew that cancer could make them more susceptible to COVID-19 and 64 $(73 \%)$ faced no delay in surgical management. Only half $(n=42$, $48 \%$ ) of respondents felt comfortable in telephonic and video consultations. Majority ( $83 \%$ ) of the patients and attendants followed all measures to decrease risk however $10(11 \%)$ respondents contacted COVID-19 during hospital stay. Conclusion: Cancer patients in our country are more worried about disease progression than severe acute respiratory syndrome-coronavirus-2. Awareness about COVID-19 is not satisfactory however most follow COVID appropriate behavior to decrease transmission risk. A majority did not feel any delay in their surgical management.

Poster (M11)

Preinvasive Disease of Cervix, Vulva, and Vagina

https://doi.org/10.3802/jgo.2021.32.S1.M11

\section{Endocervicoscopy for evaluation of transformation zone in cases of incompletecolposcopy}

\section{Guneet Kaur, Vijay Zutshi, ' Sachin Kolte}

Vardhman Mahavir Medical College and Safdarjung Hospital, New Delhi, India (drvzutshi@gmail.com)

Objective: To evaluate the role of endocervicoscopy for visualization of transformation zone (TZ) in incomplete colposcopy.

Methods: This descriptive analytical study was carried out between October 2019 and March 2021 (18 months) after obtaining ethics committee approval. A total of 92 women were recruited in the study after obtaining a written informed consent. All screen positive women and those having unhealthy looking cervix on colposcopy 
had TZ 3, were included in this study. Endocervicoscopy was performed in all women by vaginoscopic approach, using a 4-mm continuous flow hysteroscope. The endocervical curettage was taken in all cases and sent for histopathological examination and patients were managed as per the histopathology report. Sensitivity, specificity, PPV, NPV of colposcopy and endocervicoscopy was calculated. The value of $\mathrm{p}<0.05$ was considered statistically significant.

Results: The mean age was $43.76 \pm 13.02$ years and most women had parity $\geq 3(48.9 \%)$. Endocervicoscopy has a success rate of $100 \%$ for visualization of TZ completely. There were 29 women where colposcopy was normal but 5 out of these 29 women $(17.2 \%)$ had findings on endocervix. Out of these 5women, 3 were screen positive on pap smear (1 LSIL, 1 SCC, 1 AGC) and 2 had unhealthy looking cervix. In total there were two skip lesions in our study. The sensitivity, specificity, PPV, NPV and diagnostic accuracy of colposcopy was 95.6\%, 56.5\%, 68.7\%, $92.8 \%, 76.1 \%$ and endocervicoscopy was $100 \%, 80.5 \%, 58.8 \%$, $100 \%, 84.8 \%$ respectively.

Conclusion: Endocervicoscopy is a reliable method to detect the TZ inpatients with $\mathrm{TZ}$ type 3 with a success rate of $100 \%$ and a short learning curve. Skip lesions can be detected and the precise localization of the lesions allows for the depth of cone excision to be tailored, thus leading toa more conservative treatment and preserving the future fertility of women.

Poster (M12)

Surgical Techniques \& Perioperative Management

https://doi.org/10.3802/jgo.2021.32.S1.M12

\section{Laparoscopic para-aortic lymphadenectomy}

T Subramanyeshwar Rao, R. Rajagopalan Iyer," Naren Bolineni, Ajesh Raj Saxena, Srijan S Shukla

Basavatarakam Indo American Cancer Hospital \& Research Institute, Telangana, India (rajagopalan99@hotmail.com)

Objective: The primary study objective was to evaluate the safety and short-term outcomes of minimal access para-aortic lymphadenectomy.

Methods: From January 2012 and October 2020, a total of 106 patients underwent laparoscopic para-aortic lymphadenectomy. The data was analyzed through a prospectively maintained database. All laparoscopic para-aortic lymph node dissection (PALND) were performed upto the left renal vein. The procedures were done with Karl Storz 3D/Hi-Definition (HD) or Stryker 4K 1688 laparoscopic systems.

Results: The median age was 56 years (range, 11-75 years), median body mass index of $27.59 \mathrm{~kg} / \mathrm{m}^{2}$ (range, 13.67-42.19). The median operative time was 310 minutes (range, 180-430 minutes). Median intensive care unit stay was 1 day (range, 1-5 days) with a hospital stay of 6 days (4-18 days). Five patients required repair of a rent in the inferior vena cava (IVC), 4 of these were managed with laparoscopic suturing without conversion to laparotomy, while 1 patient required a laparotomy for suturing the IVC rent. Postoperatively 5 patients had chylous output in the abdominal drain, 3 of them settled with conservative management (medium chain triglyceride diet and octreotide injection), 1 patient each required ultrasound guided drain placement under anesthesia and required re-exploration. Four patients had paralytic ileus, while 1 patient each had SAIO/PTE/ $\mathrm{UTI} /$ portocaval thrombosis. Two patients required re-admission, there were no 30-day post-operative deaths. The median number of para-aortic lymph nodes dissected was 10 (range, 2-52). Conclusion: PALND when indicated in gynecologic malignancies can be safely performed with a standard two-stage 6-ports technique.

Poster (M13)

Rare Tumors \& Metastatic Tumors

https://doi.org/10.3802/jgo.2021.32.S1.M13

\section{Isolated inguinal lymphadenopathy in recurrent ovarian carcinoma: a case series}

\section{Pratima R, ${ }^{*}$ Rupinder Sekhon, Amita Naithani}

Rajiv Gandhi Cancer Institute and Research Centre, Delhi, India (dr.pratima.raj@gmail.com)

Objective: The abdominal, pelvic, and retroperitoneal cavities represent the most common sites of ovarian cancer recurrence, with isolated inguinal node involvement rarely reported. The treatment of isolated inguinal recurrence of ovarian cancer is debated, and current therapies include secondary cytoreductive surgery, chemotherapy, immunotherapy or any combination of the above. To describe the characteristics of women presenting with isolated groin recurrences.

Methods: A retrospective case series of patients with isolated inguinal lymph node recurrence were identified, patient demographic, clinical characteristics and disease course were retrieved from electronic medical record.

Results: Three cases of inguinal lymph node recurrence of ovarian cancer were identified. Mean age 50, 2 patients had a serous component, 1 had grade 3 endometroid component, stage at initial diagnosis ranged from FIGO stage IC to III. All 3 were primary recurrences in the inguinal nodal region. The progression-free survival duration from chemotherapy completion to diagnosis of inguinal recurrence varied from (12 to 24 months). 2 were treated chemotherapy f/b chemotherapy response score (CRS), $n=1$ was treated with CRS 